\title{
Effects of human collagen $\alpha-1$ type I-derived proteins on collagen synthesis and elastin production in human dermal fibroblasts
}

\author{
Su Jin Hwang ${ }^{1, \#}$, Su Hwan Kim ${ }^{2, \#}$, Woo-Young Seo ${ }^{1}$, Yelin Jeong ${ }^{3,4}$, Min Cheol Shin ${ }^{3,4}$, Dongryeol Ryu ${ }^{5}$, Sang Bae Lee , \\ Young Jin Choi $i^{2,7,8, *}$ \& KyeongJin Kim ${ }^{3,4, *}$ \\ ${ }^{1}$ ABIOTECH Co., Ltd., Suwon $16675,{ }^{2}$ Department of Agricultural Biotechnology, Seoul National University, Seoul 08826, ${ }^{3}$ Department of \\ Biomedical Sciences, College of Medicine, Inha University, Incheon 22212, ${ }^{4}$ Program in Biomedical Science \& Engineering, Inha University, \\ Incheon 22212, ${ }^{5}$ Department of Molecular Cell Biology, Sungkyunkwan University School of Medicine, Suwon 16419 , ${ }^{6}$ Division of Life \\ Sciences, Jeonbuk National University, Jeonju 54896, 'Center for Food and Bioconvergence, Seoul National University, Seoul 08826, \\ ${ }^{8}$ Research Institute for Agriculture and Life Sciences, Seoul National University, Seoul 08826, Korea
}

\begin{abstract}
Collagen type $I$ is the most abundant form of collagen in human tissues, and is composed of two identical $\alpha-1$ type I chains and an $\alpha-2$ type I chain organized in a triple helical structure. A previous study has shown that human collagen $\alpha-2$ type I (hCOL1A2) promotes collagen synthesis, wound healing, and elastin production in normal human dermal fibroblasts (HDFs). However, the biological effects of human collagen $\alpha-1$ type I (hCOL1A1) on various skin properties have not been investigated. Here, we isolate and identify the hCOL1A1-collagen effective domain (CED) which promotes collagen type I synthesis. Recombinant hCOL1A1-CED effectively induces cell proliferation and collagen biosynthesis in HDFs, as well as increased cell migration and elastin production. Based on these results, hCOL1A1-CED may be explored further for its potential use as a preventative agent against skin aging. [BMB Reports 2021; 54(6): 329-334]
\end{abstract}

\section{INTRODUCTION}

Skin aging is a common dermatological problem and is subject to intrinsic and extrinsic processes (1). Intrinsic skin aging is a physiological change, chronologically influenced by genetic and hormonal factors, whereas skin aging is extrinsically in-

${ }^{*}$ Corresponding authors. Young Jin Choi, Tel: +82-2-880-4851; Fax: +82-2-873-5695; E-mail: choiyj@snu.ac.kr; Kyeongjin Kim, Tel: +8232-860-9870; Fax: +82-32-885-8302; E-mail: kimkj@inha.ac.kr ${ }^{\#}$ These authors contributed equally to this work.

https://doi.org/10.5483/BMBRep.2021.54.6.038

Received 9 March 2021, Revised 22 March 2021, Accepted 26 April 2021

Keywords: Collagen synthesis, Elastin, hCOL1A1, Human dermal fibroblasts fluenced by environment and lifestyle factors, such as chronic light exposure and chemicals (2). These factors incorporate histopathological and immunohistochemical alternations in each skin layer, such as changes in skin appearance after UV exposure (3-5). Furthermore, both intrinsic and extrinsic factors result to decreased levels of elastin and collagen synthesis in fibroblasts and increased melanin production in melanocytes (6-8).

Collagen and elastin are structural proteins in the skin that maintain both elasticity and firmness (9). Interestingly, the fibrous protein collagen constitutes the majority of the skin and contributes to maintain mechanical strength of tissues (10). The collagen molecule is composed of three chains wound together in a tight triple helix. Every third amino acid is glycine that fits perfectly inside the helix. The triple helix is mainly composed of glycine, followed by proline (or hydroxyproline), and then alanine residues (11). Fibroblasts that produce both collagen and the elastin matrix can be induced by glycolic acid, and these distinctive fibroblast cells are observed in the papillary level of the second layer of the dermis (9). Together, collagen has a specific amino acid composition, which is further associated with fibrogenesis.

Collagen type I, the most abundant type of collagen in humans, is a major structural protein dominant in the bone (more than $90 \%$ of the organic mass), tendons, ligaments, cornea, and many interstitial connective tissues (11). Different types of collagen vary in the primary amino acid sequences of their polypeptide chains. Structurally, type I collagen comprises two identical $\alpha-1$ type I chains and an $\alpha-2$ type I chain organized in a triple helix (11).

Migration of human dermal fibroblasts (HDFs) is essential for skin wound healing and remodeling. The proliferation of HDFs causes their migration into the wound bed, leading to expression of thick actin bundles in myofibroblasts and the synthesis of new extracellular matrix (ECM) (12). Previous studies have identified that growth factors/cytokines such as fibroblast growth

ISSN: 1976-670X (electronic edition)

Copyright (C) 2021 by the The Korean Society for Biochemistry and Molecular Biology

(c) This is an open-access article distributed under the terms of the Creative Commons Attribution Non-Commercial License (http://creativecommons.org/licenses/by-nc/4.0) which permits unrestricted non-commercial use, distribution, and reproduction in any medium, provided the original work is properly cited. 
factors (FGF), hepatocyte growth factor (HGF), transforming growth factor- $\beta 1$ (TGF- $\beta 1$ ), epidermal growth factor (EGF), platelet-derived growth factor (PDGF), and insulin-like growth factor (IGF) are directly or indirectly associated with HDF motility (12-14). The impact of these growth factors on HDFs covers a broad range of biological phenotypes, such as cellular proliferation, regeneration, and metabolism (15-17).

Our previous study has shown that human collagen alpha-2 type I (hCOL1A2) promotes collagen synthesis, elastin production, and wound healing in normal dermal fibroblasts (18). Our analysis has identified that the hCOL1A2-derived peptide promotes the improvement of skin properties compared with the effects of marine collagen. Although human collagen type I is composed of a triple helix of human collagen alpha- 1 type I (hCOL1A1) wound together with hCOL1A2 (11), the effects of hCOL1A1 on skin properties have not been investigated. In this study, we found that hCOL1A1-derived proteins promote collagen synthesis, elastin production, and cell migration in HDFs and HaCaT keratinocytes.

\section{RESULTS AND DISCUSSION}

\section{Identification of the efficiency of hCOL1A1 domains in collagen type I synthesis}

Previous studies have suggested that collagen type I synthesis and fibroblast cell proliferation are required to maintain the strength and elasticity of the skin (19-21). We have previously reported that hCOL1A2-derived proteins promote collagen type I synthesis, elastin production, and fibroblast cell proliferation in normal HDFs (18). To investigate the biological function of hCOL1A1 - another major component of human collagen type I, in human skin fibroblasts-we examined the effects of hCOL1A1 domains on collagen type I synthesis in normal HDFs, similar to our previous study (18). Full-length hCOL1A1 and various deletion mutants were generated (Fig. 1A), and Normal HDFs were transfected with plasmids encoding hemagglutinin (HA) (negative control; NC), HA-tagged full-length COL1A1 (FL; chain domain), and the deletion mutants, namely of the $\mathrm{N}$-terminal $(\mathrm{N})$, middle $(\mathrm{M})$, and $\mathrm{C}$-terminal chain $(\mathrm{C})$. The expression of hCOL1A1 FL and deletion mutants was analyzed by immunoblotting (Fig. 1B), and the levels of collagen type I were measured using an hCOL1A1 enzyme-linked immunosorbent assay (ELISA) kit. Interestingly, the $\mathrm{M}$ deletion mutant significantly induced collagen type I synthesis compared with the NC or other deletion mutants (Fig. 1C).

In order to narrow down the protein region in the M-domain critical for collagen type I synthesis, we further constructed several deletion mutants inside the M-domain. HDFs were transfected with plasmids encoding $\mathrm{HA}(\mathrm{NC})$ and the deletion mutants, namely small middle A (SMA), small middle B (SMB), small middle C (SMC), small middle D (SMD), and small middle $\mathrm{E}$ (SME). Cells were analyzed after $48 \mathrm{~h}$ of transfection using immunoblotting (Fig. 1D) and the amount of collagen type I was measured with an hCOL1A1 ELISA kit. The SME
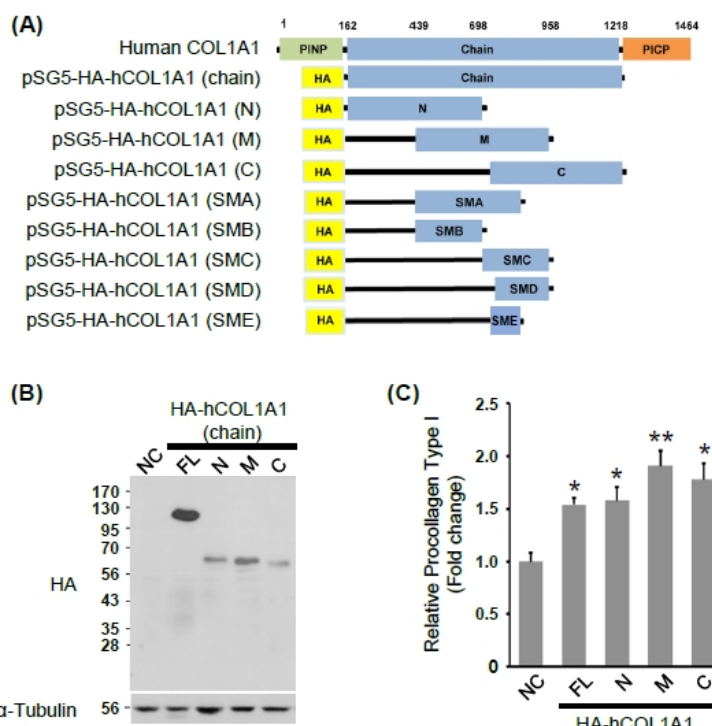

(D)

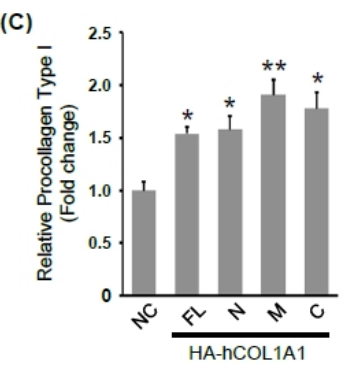

(E)
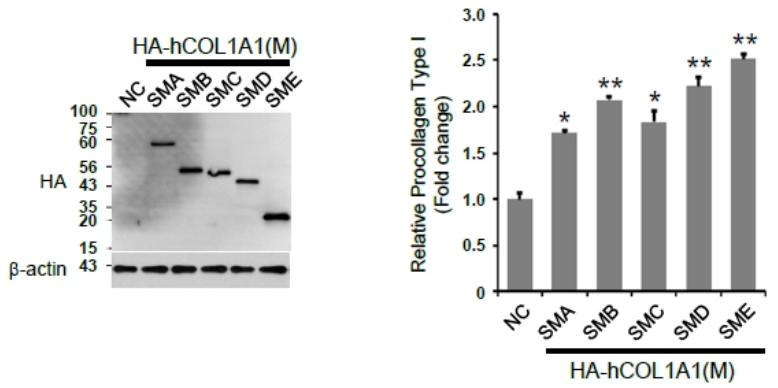

Fig. 1. Identification of hCOL1A1 domain for the synthesis of collagen type I. (A) Schematic representing full-length hCOL1A1 and its various deletion mutants. (B and $\mathrm{C}$ ) Human dermal fibroblasts (HDFs) were transfected with plasmids encoding hemagglutinin (HA), as the negative control (NC), HA-tagged full length hCOL1A2 (FL), and each of the deletion mutants ( $\mathrm{N}$-terminal chain $[\mathrm{N}]$, middle chain [M], or C-terminal chain [C]). Forty-eight hours after transfection, cells were collected and cell lysates were analyzed by immunoblotting with anti-HA and anti-tubulin antibodies (B). The amount of collagen type I in transfected HDF culture media was measured with an enzyme-linked immunosorbent assay (ELISA) kit (C). (D and E) HDF cells were transfected with plasmids encoding $\mathrm{HA}(\mathrm{NC})$ and each of the indicated mutants (small middle A [SMA], small middle B [SMB], small middle C [SMC], small middle $\mathrm{D}[\mathrm{SMD}]$, or small middle $\mathrm{E}$ [SME]). Forty-eight hours after transfection, cells were collected and cell lysates were analyzed by immunoblotting with anti-HA and anti-tubulin antibodies (D). The amount of collagen type I was measured with an ELISA kit in a transfected HDF-cultured media (E). Results are presented as mean $\pm \mathrm{SD}$ of three independent experiments. Student's $t$-test was used for statistical analyses (*P $<0.05$, **P $<0.005)$.

deletion mutant significantly increased collagen type I synthesis compared with the other deletion mutants; however, the other mutants (SMA, SMB, SMC, or SMD) were also capable of increasing collagen type I synthesis (Fig. 1E). Thus, the C-ter- 
minal region spanning amino acid residues 743-880 of SMA and overlapping with both SMC and SMD is the functional and effective region of hCOL1A1 that affects collagen type I synthesis. Then, the SME was further designated as hCOL1A1collagen effective domain (hCOL1A1-CED).

\section{Isolation and identification of recombinant hCOL1A1-CED protein}

Next we cloned a histidine (His)-tagged hCOL1A1-CED into the pET-28a (His-tag) vector. Recombinant proteins were produced by the Escherichia coli Rosetta2 (DE3) strain and purified as previously represented (22). To ensure the molecular composition ratio, purified hCOL1A1-CED was isolated and visualized as a single band of approximately 21 kDa by SDS-PAGE, as also shown the uninduced or induced TCP (total cell proteins) (Fig. 2A). Then, hCOL1A1-CED was confirmed using an anti-His antibody (Fig. 2B) as well as an anti-COL1A1 (CED-specific) antibody (Fig. 2C).

To further confirm the sequences of purified recombinant hCOL1A1-CED, we analyzed the peptides from in-gel digestion with trypsin using liquid chromatography-mass spectrometry (LC/MS), showing a representative MS/MS spectrum for the identified peptides originating from hCOL1A $1\left({ }^{781}\right.$ GESGPSGPA GPTGAR $^{796}, 1297.61724 \mathrm{~m} / \mathrm{z}$ ) in Fig. 2D and Supplementary Table 1.

\section{Effects of recombinant hCOL1A1-CED protein on cell proliferation and collagen biosynthesis}

We compared the effects of hCOL1A1-CED and EGF on HDF cell proliferation, as these have previously been reported to promote cell proliferation $(8,18,23)$. As shown in Fig. 3A, both hCOL1A1-CED protein $(10 \mu \mathrm{g} / \mathrm{ml})$ and EGF $(1 \mu \mathrm{g} / \mathrm{ml})$ showed similar HDF cell proliferation (Fig. $3 \mathrm{~A}$ ). TGF- $\beta 1$ has been reported to significantly increase collagen type I synthesis and proliferation in both embryonic pulmonary fibroblasts and HDFs in a crowded cell culture system $(8,24)$. Therefore, to further investigate the effects of hCOL1A1-CED, we compared its effect with that of TGF- $\beta 1$ or EGF on collagen synthesis. We determined the amount of collagen type I secreted in the culture media of HDFs stimulated with TGF- $\beta 1(10 \mathrm{ng} / \mathrm{ml})$, EGF $(1 \mu \mathrm{g} / \mathrm{ml})$, or hCOL1A1-CED $(10 \mu \mathrm{g} / \mathrm{ml})$. We found that hCOL1A1-CED $(10 \mu \mathrm{g} / \mathrm{ml})$, TGF- $\beta 1(10 \mathrm{ng} / \mathrm{ml})$ and EGF $(1 \mu \mathrm{g} /$ $\mathrm{ml}$ ) showed similar increases in collagen type I synthesis induction, endogenous Col1a1 gene expression and fibroblast cell proliferation (Fig. 3B-D).

Furthermore, hCOL1A1-CED was detectable in HDF cell lysates even after the culture medium treated with hCOL1A1-CED was washed out (Fig. 3E), suggesting that hCOL1A1-CED is bioactive, similar to other hydrolyzed collagen peptides, and can be absorbed and utilized by HDFs. Together, these results demonstrate that hCOL1A1-CED significantly increases collagen type I synthesis and fibroblast proliferation.
(A)

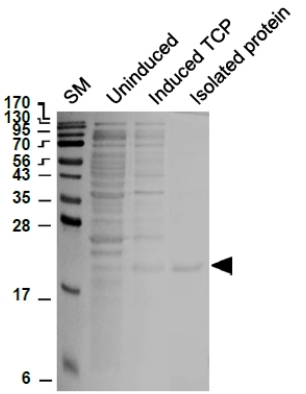

(D)

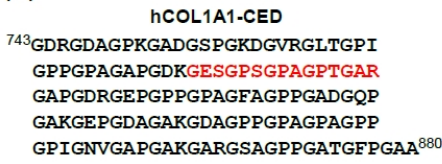

(B)

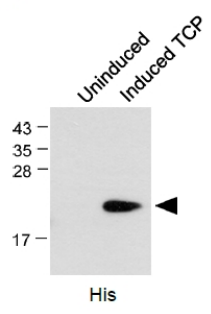

(C)

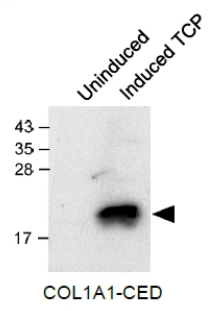

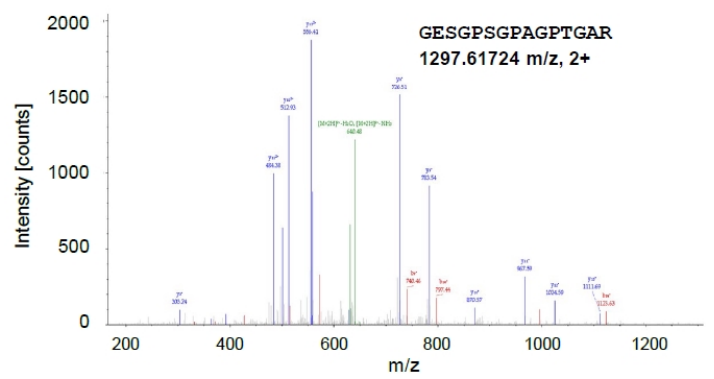

Fig. 2. Identification of purified hCOL1A1-CED. (A) Coomassie Blue staining of uninduced (without IPTG), induced TCP (total cell protein), or isolated hCOL1A1-CED showed a prominent band; arrows indicate that hCOL1A1-CED was identified using (B) anti-His and (C) antihCOL1A1 antibodies by western blot analysis. (D) Identification of hCOL1A1-CED protein in the gel band of isolated hCOL1A1-CED by liquid chromatography-mass spectrometry. The panels show the representative MS/MS spectrum for the identified peptides of GESGPSGPAGPTGAR $(1297.61724 \mathrm{~m} / \mathrm{z}, 2+)$. 


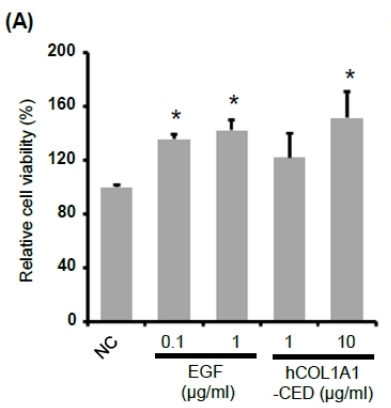

(D)
(B)

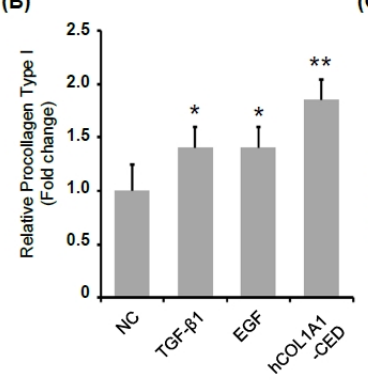

(C)

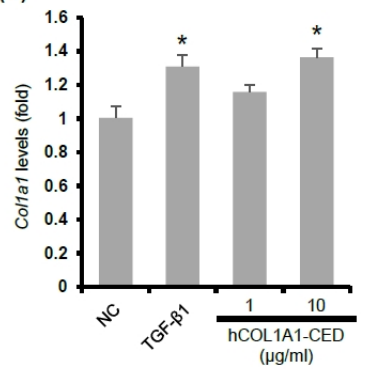

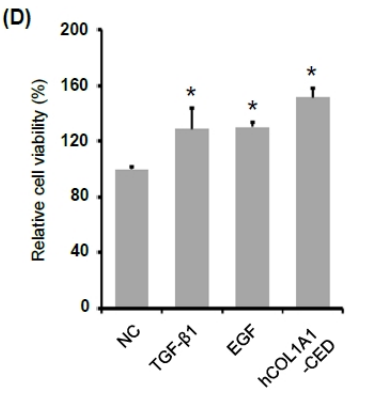

(E)
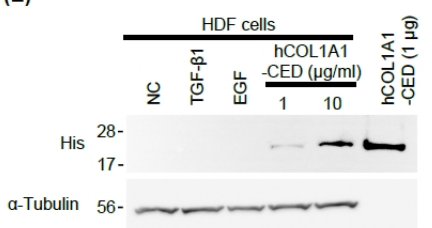

a-Tubulin 56 -

Fig. 3. hCOL1A1-CED stimulates cell proliferation and collagen synthesis in HDFs. (A) HDF cells were treated with EGF (0.1 and $1 \mu \mathrm{g} / \mathrm{ml})$ and hCOL1A1-CED (1 and $10 \mu \mathrm{g} / \mathrm{ml})$ for $48 \mathrm{~h}$. Cell proliferation, induced by EGF and hCOL1A1-CED, was determined by 3-(4,5-dimethylthiazol2-yl)-2,5-diphenyltetrazolium bromide (MTT) assay. (B and C) HDF cells were treated with EGF (1 $\mu \mathrm{g} / \mathrm{ml} ; 166.7 \mathrm{nM}), \mathrm{hCOL1A} 1-\mathrm{CED}(10 \mu \mathrm{g} / \mathrm{ml}$; $840.9 \mathrm{nM})$, or TGF- $\beta 1(10 \mathrm{ng} / \mathrm{ml} ; 781.3 \mathrm{pM})$ for $48 \mathrm{~h}$. The amount of collagen type I in the HDF culture media and mRNA levels of Col1a1 in HDF cells were measured by enzyme-linked immunosorbent assay (B) or qRT-PCR (C), respectively. Cell proliferation was determined by MTT assay (D). (E) HDF cells were treated with EGF $(1 \mu \mathrm{g} / \mathrm{ml})$, TGF- $\beta 1(10 \mathrm{ng} / \mathrm{ml})$, or hCOL1A1-CED (1 and $10 \mu \mathrm{m} / \mathrm{ml})$, for $48 \mathrm{~h}$. Cells were collected $48 \mathrm{~h}$ post-treatment and cell lysates were analyzed by immunoblotting with anti-His (upper panel) or anti- $\alpha$-tubulin antibodies, respectively. Results are presented as the mean \pm standard deviation of three independent experiments. Student's $t$-test was used for statistical analyses $(* \mathrm{P}<0.05, * * P<0.005)$.

\section{Effects of recombinant hCOL1A1-CED protein on migration and elastin production}

Previous studies have determined that the abundant amino acid residues in collagen peptides support cellular growth and proliferation (25). We further investigated the effects of hCOL 1A1-CED on cell adhesion and growth using the human keratinocyte cell line $\mathrm{HaCaT}$, which is an in vitro model of skin wound healing (26). We tested the effects of TGF- $\beta 1$, EGF, or hCOL1A1-CED in the transwell migration assay using $\mathrm{HaCaT}$ cell monolayers and the scratch-wound healing assay using HaCaT and HDF cells, and as shown in Fig. 4A, B, hCOL1A1CED treatment significantly increased cell migration, compared with the NC.

Previous studies have suggested that the skin aging is induced by intrinsic and extrinsic pathway, which are both accompanied with histopathological and immunohistochemical alternations (1). Intrinsic pathway of skin aging is a process of both the qualitative and quantitative changes, including diminished or defective collagen and elastin production in the dermis (27, 28). To evaluate the effect of hCOL1A1-CED on elastin synthesis, we analyzed elastin levels in the culture media of HDFs treated with TGF- $\beta 1(10 \mathrm{ng} / \mathrm{ml})$, EGF $(1 \mu \mathrm{g} / \mathrm{ml})$, or hCOL1A1-
CED $(10 \mu \mathrm{g} / \mathrm{ml})$. Elastin synthesis significantly increased in the hCOL1A1-CED-treated group, compared with the TGF- $\beta 1$ (10 $\mathrm{ng} / \mathrm{ml})$ and EGF $(1 \mu \mathrm{g} / \mathrm{ml})$-treated groups and the NC group, whereas fibronectin synthesis was not affected by hCOL1A1-CED treatment (Fig. 4C, D), likely representing that the lack of binding affinity to fibronectin (29) does not speculate on fibronectin synthesis.

Next, to test whether hCOL1A1-CED is associated with skin aging, we further measured melanin synthesis. We observed that melanin synthesis was not altered by treatment with hCOL1A1-CED in B16F10 melanoma cells, whereas vitamin C treatment significantly reduced melanin synthesis (Fig. 4E). Additionally, the gene expression of matrix metalloproteinase 1 (Mmp1), Mmp2, Mmp3, or tissue inhibitor of metalloproteinase 1 (Timp1) was significantly downregulated by hCOL1A1-CED treatment in HDF cells (Fig. 4F), which is consistent with previous results that these changes are associated with aging and collagen degradation (30). Together, these results demonstrate that hCOL1A1-CED induces cell migration and elastin synthesis, but not fibronectin synthesis.

In conclusion, hCOL1A1-derived CED enhances collagen type I and elastin synthesis, cell proliferation as well as cell 

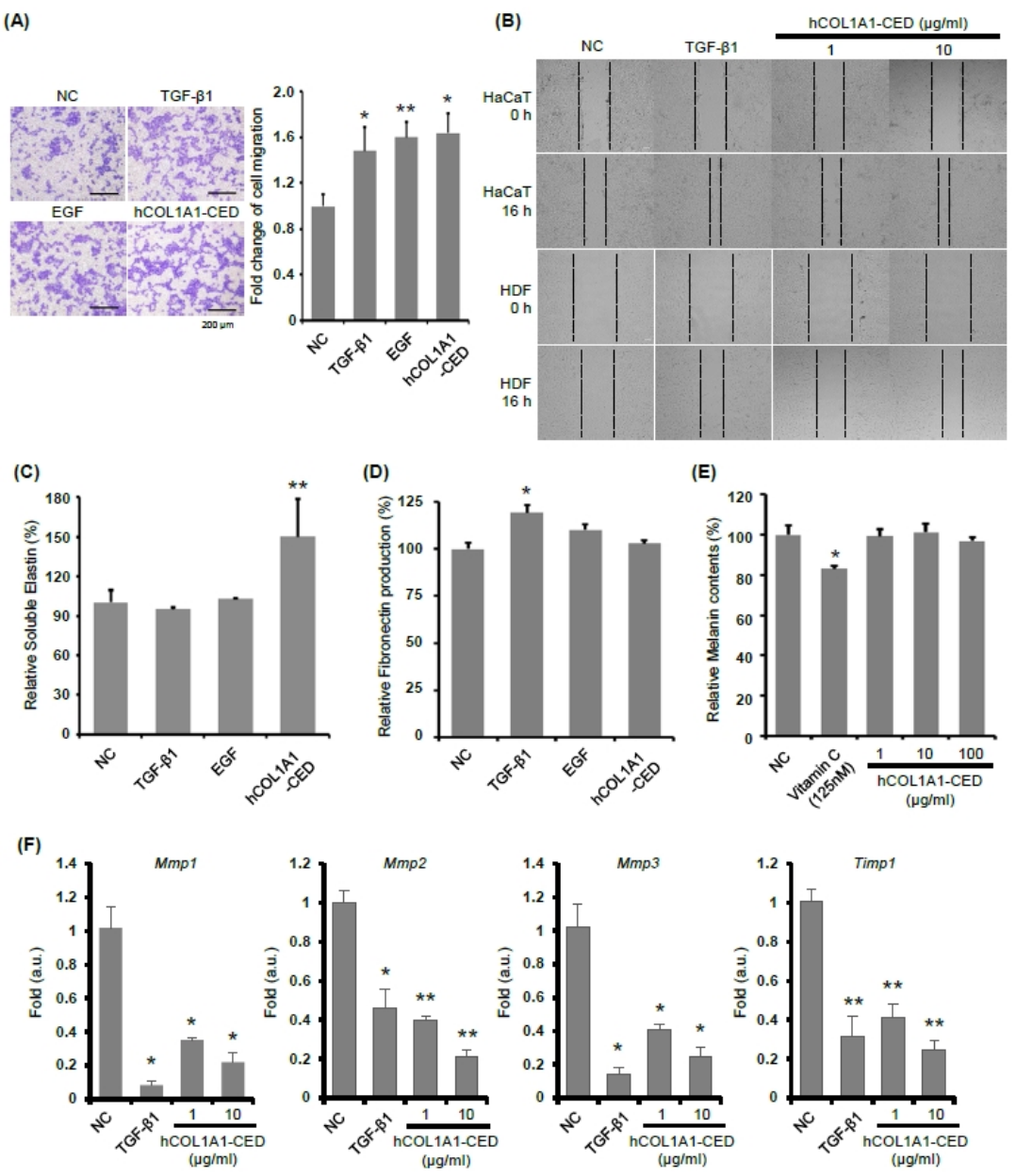

Fig. 4. hCOL1A1-derived protein increases the migration efficiency and elastin production, but not fibronectin production. (A) HaCaT cells were treated with TGF- $\beta 1(10 \mathrm{ng} / \mathrm{ml})$, EGF $(1 \mu \mathrm{g} / \mathrm{ml})$, or hCOL1A1- CED $(10 \mu \mathrm{g} / \mathrm{ml})$ and then subjected to transwell migration assays. Migration was monitored for up to $20 \mathrm{~h}$. The same fields were photographed immediately after treatment and after $20 \mathrm{~h}$, and images were superimposed using Adobe Photoshop. Areas were measured using Image J. A representative result is shown. (B) In vitro wound healing assay in HaCaT (top) or HDF (bottom) cells with TGF $\beta-1(10 \mathrm{ng} / \mathrm{ml})$ or hCOL1A1-CED at the indicated concentration. The cells were photographed immediately after wounding and 16 hours later, and the pictures were superimposed. A representative result is shown. (C and D) HDF cells were treated with TGF- $\beta 1(10 \mathrm{ng} / \mathrm{ml})$, EGF $(1 \mu \mathrm{g} / \mathrm{ml})$, and hCOL1A1-CED (10 $\mu \mathrm{g} / \mathrm{ml})$ for $48 \mathrm{~h}$. The amount of elastin (C) and fibronectin (D) in the HDF culture media was measured using enzyme-linked immunosorbent assay. (E and F) Melanin contents in B16F10 melanoma cells (E) or expression of indicated genes in HDF cells (F) after treatment with TGF $\beta-1$ or hCOL1A1. Results are presented as the mean \pm standard deviation of three independent experiments. Student's t-test was used for statistical analyses $(* \mathrm{P}<0.05$, $* * P<$ 0.005).

migration; both of these proteins are known to be effective anti-skin aging agents. Although the hCOL1A1-derived CED could induce anti-wrinkle effects, such as those facilitated by the migration and proliferation of fibroblasts, we should remain cognizant of its potential side effects when used as a cosmeceutical ingredient.

\section{MATERIALS AND METHODS}

(The detailed methods are described in the "Supplementary
Materials and Methods".)

\section{ACKNOWLEDGEMENTS}

This work was supported by an INHA UNIVERSITY Research Grant to K.K., research funds for newly appointed professors of Jeonbuk National University to S.B.L., and National Research Foundation of Korea (NRF) grants funded by the Korean government (MSIT) to K.K. (2020R1C1C1004015) and S.B.L. (2020R1C1C1014281). 


\section{CONFLICTS OF INTEREST}

The authors have no conflicting interests.

\section{REFERENCES}

1. El-Domyati M, Attia S, Saleh F et al (2002) Intrinsic aging vs. photoaging: a comparative histopathological, immunohistochemical, and ultrastructural study of skin. Exp Dermatol 11, 398-405

2. Mancini M, Lena AM, Saintigny G et al (2014) MicroRNAs in human skin ageing. Ageing Res Rev 17, 9-15

3. Ganceviciene R, Liakou Al, Theodoridis A, Makrantonaki E and Zouboulis CC (2012) Skin anti-aging strategies. Dermatoendocrinol 4, 308-319

4. Rendon MI and Gaviria JI (2005) Review of skin-lightening agents. Dermatol Surg 31, 886-889; discussion 889

5. Petit L and Pierard GE (2003) Skin-lightening products revisited. Int J Cosmet Sci 25, 169-181

6. Kim WS, Park BS, Park SH, Kim HK and Sung JH (2009) Antiwrinkle effect of adipose-derived stem cell: activation of dermal fibroblast by secretory factors. J Dermatol Sci 53, 96-102

7. Kim J, Kang S, Kwon H, Moon H and Park MC (2019) Dual functional bioactive-peptide, AIMP1-derived peptide (AdP), for anti-aging. J Cosmet Dermatol 18, 251-257

8. Edgar S, Hopley B, Genovese L, Sibilla S, Laight D and Shute J (2018) Effects of collagen-derived bioactive peptides and natural antioxidant compounds on proliferation and matrix protein synthesis by cultured normal human dermal fibroblasts. Sci Rep 8, 10474

9. Krutmann J (2011) [Skin aging/anti-aging strategies]. Hautarzt 62,576

10. Gelse K, Poschl E and Aigner T (2003) Collagens - structure, function, and biosynthesis. Advanced Drug Delivery Reviews 55, 1531-1546

11. Gelse K, Poschl E and Aigner T (2003) Collagens-structure, function, and biosynthesis. Adv Drug Deliv Rev 55, 15311546

12. Singer AJ and Clark RA (1999) Cutaneous wound healing. N Engl J Med 341, 738-746

13. Imanishi J, Kamiyama K, Iguchi I, Kita M, Sotozono $C$ and Kinoshita S (2000) Growth factors: importance in wound healing and maintenance of transparency of the cornea. Prog Retin Eye Res 19, 113-129

14. Park JS, Kim JY, Cho JY, Kang JS and Yu YH (2000) Epidermal growth factor (EGF) antagonizes transforming growth factor (TGF)-beta1-induced collagen lattice contraction by human skin fibroblasts. Biol Pharm Bull 23, 1517-1520

15. Van Cauter E and Plat L (1996) Physiology of growth hormone secretion during sleep. J Pediatr 128, S32-37

16. Bartke A (2008) Growth hormone and aging: a challenging controversy. Clin Interv Aging 3, 659-665

17. Sonntag WE, Csiszar A, deCabo R, Ferrucci $L$ and Ungvari Z (2012) Diverse roles of growth hormone and insulin-like growth factor-1 in mammalian aging: progress and controversies. J Gerontol A Biol Sci Med Sci 67, 587-598

18. Hwang SJ, Ha GH, Seo WY, Kim CK, Kim K and Lee SB (2020) Human collagen alpha-2 type I stimulates collagen synthesis, wound healing, and elastin production in normal human dermal fibroblasts (HDFs). BMB Rep 53, 539-544

19. Trojanowska M, LeRoy EC, Eckes B and Krieg T (1998) Pathogenesis of fibrosis: type 1 collagen and the skin. J Mol Med (Berl) 76, 266-274

20. Chiquet $M$, Matthisson $M$, Koch $M$, Tannheimer $M$ and Chiquet-Ehrismann R (1996) Regulation of extracellular matrix synthesis by mechanical stress. Biochem Cell Biol 74, 737-744

21. Majora M, Wittkampf T, Schuermann B et al (2009) Functional consequences of mitochondrial DNA deletions in human skin fibroblasts: increased contractile strength in collagen lattices is due to oxidative stress-induced lysyl oxidase activity. Am J Pathol 175, 1019-1029

22. Seo WY, Kim JH, Baek DS et al (2017) Production of recombinant human procollagen type I C-terminal propeptide and establishment of a sandwich ELISA for quantification. Sci Rep 7, 15946

23. Andl CD, Mizushima T, Nakagawa $\mathrm{H}$ et al (2003) Epidermal growth factor receptor mediates increased cell proliferation, migration, and aggregation in esophageal Keratinocytes in vitro and in vivo. J Biol Chem 278, 1824-1830

24. Chen CZ, Peng YX, Wang ZB et al (2009) The Scar-in-a-Jar: studying potential antifibrotic compounds from the epigenetic to extracellular level in a single well. $\mathrm{Br}$ J Pharmacol 158, 1196-1209

25. Hu Z, Yang P, Zhou CX, Li SD and Hong PZ (2017) Marine collagen peptides from the skin of nile tilapia (oreochromis niloticus): characterization and wound healing evaluation. Mar Drugs 15, 102

26. Walter MNM, Wright KT, Fuller HR, MacNeil $S$ and Johnson WE (2010) Mesenchymal stem cell-conditioned medium accelerates skin wound healing: an in vitro study of fibroblast and keratinocyte scratch assays. Exp Cell Res 316, 1271-1281

27. Quan T, Shao Y, He T, Voorhees JJ and Fisher GJ (2010) Reduced expression of connective tissue growth factor (CTGF/CCN2) mediates collagen loss in chronologically aged human skin. J Invest Dermatol 130, 415-424

28. Seite S, Zucchi H, Septier D, Igondjo-Tchen S, Senni K and Godeau G (2006) Elastin changes during chronological and photo-ageing: the important role of lysozyme. J Eur Acad Dermatol Venereol 20, 980-987

29. Erat MC, Slatter DA, Lowe ED et al (2009) Identification and structural analysis of type I collagen sites in complex with fibronectin fragments. Proc Natl Aca Sci U S A 106, 4195-4200

30. Freitas-Rodriguez S, Folgueras AR and Lopez-Otin C (2017) The role of matrix metalloproteinases in aging: Tissue remodeling and beyond. Biochim Biophys Acta Mole Cell Res 1864, 2015-2025 